\title{
XI CONGRESO \\ DEL PARTIDO COMUNISTA DE ESPAÑA
}

Entre los días 14 y 18 de diciembre de 1983 tuvo lugar el undécimo Congreso de los comunistas españoles. Durante cinco días 810 delegados (véase tabla I) - de los que alrededor de un 77 por 100 ingresaron en el Partido antes de 1976, cerca de la mitad eran obreros industriales y casi la totalidad hombres (tabla II) - - se iban a enfrentar a la más grave de las crisis atravesada por el PCE desde su fundación, según reconocerían sus propios dirigentes. En términos organizativos, la crisis se manifestaba en un fuerte descenso del número de afiliados: de 201.740 en abril de 1978 a 84.652 en diciembre de 1983 (véase tabla III). No de forma menos espectacular se había expresado la crisis en el ámbito electoral: en las elecciones generales de 1982 se perdieron algo más de un millón de votos con respecto de los alcanzados en 1979 (PCE+PSUC). Y ya en términos políticos, los desacuerdos en el seno del Partido, que venían manifestándose desde mucho tiempo atrás, cristalizaron definitivamente - durante el proceso de preparación del Congreso- en dos posiciones que iban a aparecer como alternativas en prácticamente todos los temas objeto de debate (política internacional, valoración de la coyuntura política, política frente al Partido del Gobierno y modelo de Partido).

Los dos bloques en los que aparecía dividido el Congreso se aglutinaban uno en torno al secretario general, Gerardo Iglesias, quien además contaba con el apoyo de la mayoría del Comité Central, y otro alrededor de Santiago Carrillo, en él se encontraba la mayoría de las organizaciones de Madrid (prácticamente la totalidad: 102 delegados frente a 1 ; no obstante, hay que tener en cuenta que en esta organización se aplicó un sistema mayoritario para la elección de los delegados), Castilla y León, Castilla-La Mancha, Galicia y País Valenciano, así como minorías de las restantes organizaciones. Se incluían en este grupo, además, alrededor de 30 de los 93 miembros por elección que formaban parte del Comité Central saliente. Está por determinar en este segundo bloque la homogeneidad real —más allá de necesidades tácticas durante el Congreso- entre las posiciones mantenidas por Santiago Carrillo y las sostenidas por los sectores abiertamente opuestos a la política de Gerardo Iglesias. En numerosas ocasiones, estos últimos cuestionaron muchas de las posiciones decididamente defendidas en el pasado por el anterior secretario general.

Finalmente, las tesis aprobadas fueron las presentadas por el Comité Central después de la incorporación de numerosas enmiendas parciales en los debates que tuvieron lugar en las comisiones. Pese al triunfo de las tesis oficiales, los resultados oficiales reflejaban que el PCE seguía encontrándose con una importante división en su seno tras la celebración del Congreso. En páginas posteriores recogemos un extracto de las tesis aprobadas junto a algunas de las críticas principales esgrimidas por los defensores de las tesis alternativas en sus intervenciones en los plenos. Recogemos, igualmente, el resultado de las votaciones de cada una de las tesis. 
Las discrepancias entre los delegados quedaron también transparentemente reflejadas en los resultados de las votaciones para la elección del Comité Central (véase tabla IV). No obstante, la composición final de este órgano permitió que Gerardo Iglesias fuera reelegido como secretario general por 69 votos a favor contra 31. Cómoda mayoría que en principio le tendría que facilitar el desarrollo de su política para la recuperación interna y externa del Partido. Tarea esta, sin duda, de grandes dificultades no sólo por los elementos externos que la problematizan, sino también, y de manera importante, porque las discrepancias que sobre aspectos fundamentales de las concepciones políticas del Partido existían en su interior no parecen, a tenor de los resultados del Congreso, definitivamente superadas.

\section{TABLA I \\ RELACION DE DELEGADOS ASISTENTES AL XI CONGRESO ${ }^{1}$}

\begin{tabular}{|c|c|c|}
\hline Delegaciones & Número de carnets & Delegados \\
\hline Andalucía .... & 21.624 & 173 \\
\hline Aragón & 1.104 & 9 \\
\hline Asturias .......................... & 6.973 & 56 \\
\hline Baleares $\ldots \ldots \ldots \ldots \ldots \ldots \ldots$ & 832 & 7 \\
\hline Canarias ..... & 1.418 & 11 \\
\hline Castilla y León ................ & 2.249 & 18 \\
\hline Castilla-La Mancha ......... & 5.287 & 42 \\
\hline Cataluña ...................... & 9.016 & 64 \\
\hline Ceuta-Melilla ...................... & 28 & 1 \\
\hline Euskadi ...... & 2.290 & 23 \\
\hline Extremadura & 2.828 & 23 \\
\hline Cantabria ....................... & 970 & 8 \\
\hline Galicia ........................ & 1.610 & 13 \\
\hline Madrid .......................... & 12.842 & 103 \\
\hline Murcia .................... & 1.877 & 15 \\
\hline País Valenciano ............. & 10.410 & 83 \\
\hline Rioja $\ldots \ldots \ldots \ldots \ldots \ldots \ldots$ & 131 & 1 \\
\hline Francia $\ldots \ldots \ldots \ldots \ldots \ldots \ldots$ & 2.845 & 23 \\
\hline R.F.A. & 699 & 5 \\
\hline Suiza ............................. & $65+563$ & 5 \\
\hline Bélgica ......................... & 655 & 5 \\
\hline Holanda $*$........................ & 176 & 5 \\
\hline 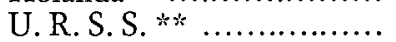 & 252 & 6 \\
\hline Ultramar $* * * \ldots \ldots \ldots \ldots$ & 215 & 5 \\
\hline Comité Central .............. & - & 92 \\
\hline Garantías y contr. ........... & - & 9 \\
\hline Control de finan. ............. & - & 5 \\
\hline Total & 84.652 & 810 \\
\hline
\end{tabular}

Holanda, Luxemburgo, Suecia, Gran Bretaña y Dinamarca.

* U. R. S. S., Checoslovaquia, R. D. A., Rumania, Polonia y Hungría.

*** Australia, Argelia, México, Cuba y Venezuela.

1 FUente: Mundo Obrero, 16 a 22 de diciembre de 1983. 


\section{TABLA II}

1. Categorias socioprofesionales de los delegados asistentes al XI Congreso

Obreros industriales

Obreros agrícolas

Campesinos

Servicios

Amas de casa

Empleados administrativos

Profesionales de la cultura

Estudiantes

Empresarios

2. Sexo

Mujeres

3. Periodos de ingreso en el Partido

Antes de 1939

Entre 1939-1950

23

Entre 1951-1960

Entre 1961-1970

Entre 1971-1976

278

Entre 1977-1980

Media de edad: 39 años.

Media de años de militancia: 14 años.

Fuente: Comisión de Credenciales del Congreso. 


\section{TABLA III \\ NUMERO DE AFILIADOS \\ AL PCE EN LA FECHA DE REALIZACION \\ DE LOS TRES ULTIMOS CONGRESOS}

\begin{tabular}{|c|c|c|c|}
\hline & IX & $\mathrm{x}$ & $\mathrm{XI}$ \\
\hline Andalucía & 39.373 & 28.680 & 21.624 \\
\hline Aragón $\ldots \ldots \ldots \ldots \ldots \ldots \ldots$ & 3.044 & 1.571 & 1.104 \\
\hline Asturias $\ldots . . . . . \ldots \ldots \ldots \ldots \ldots$. & 10.940 & 7.557 & 6.973 \\
\hline Baleares .... & 2.501 & 975 & 832 \\
\hline Canarias ... & 4.000 & 1.499 & 1.418 \\
\hline Castilla-La Mancha & 10.017 & 7.564 & 5.278 \\
\hline Castilla y León ........ & 4.599 & 3.046 & 2.249 \\
\hline Cataluña ........................ & 40.000 & 21.800 & 8.016 \\
\hline Ceuta-Melilla ................. & 45 & 37 & 28 \\
\hline Euskadi ......................... & 8.083 & 5.611 & 2.920 \\
\hline Extremadura $\ldots . . . . . . . . . . .$. & 4.286 & 2.939 & 2.828 \\
\hline 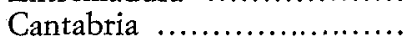 & 1.749 & 1.268 & 970 \\
\hline Galicia $\ldots \ldots \ldots \ldots \ldots \ldots \ldots$ & 4.000 & 3.050 & 1.610 \\
\hline (................... & 31.895 & 20.771 & 12.842 \\
\hline Murcia & 4.811 & 2.979 & 1.877 \\
\hline País Valenciano ............... & 22.060 & 15.509 & 10.470 \\
\hline Rioja ............................ & 387 & 238 & 131 \\
\hline Emigración ................... & 10.000 & 6.975 & 5.470 \\
\hline Total & 201.740 & 132.069 & 84.652 \\
\hline
\end{tabular}

FuENTE: Tiempo, 16 de diciembre de 1983.

TABLA IV

VOTOS OBTENIDOS POR LOS MIEMBROS ELEGIDOS PARA EL COMITE CENTRAL

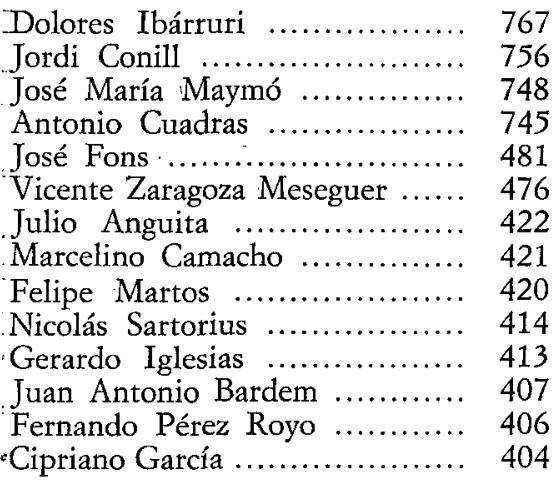

Enrique Curiel ................ 403

José López Bulla ................ 403

Julián Atiza ...................... 402

Eulalia Vintró ................... 402

Adolfo Pastor .................... 402

Leonor Bornao ................... 402

José Luis Núñez ................ 401

José Luis Buhigas ................ 401

Santiago Carrillo Solares ........ 401

Simón Sánchez Montero ........ 401

Antonio Gutiérrez ................ 400

Francisco Palero ................ 400

Ernesto Caballero ................ 400

José María Coronas .............. 399 


\begin{tabular}{|c|c|}
\hline & \\
\hline Antonio Montalbán & \\
\hline Pedro Marset & \\
\hline Juan Bosco & \\
\hline Francisco Frutos & \\
\hline Aristu & \\
\hline andoval & \\
\hline uel Ang & \\
\hline Daniel Lacalle & \\
\hline ndez ....... & \\
\hline Rom & \\
\hline co Romero Marín & \\
\hline Roney & \\
\hline do López Salinas & \\
\hline Romero & \\
\hline & \\
\hline $\mathrm{Buc}$ & \\
\hline Cámara Fernández & \\
\hline Morán & \\
\hline Iore & \\
\hline Tueros & \\
\hline to Alvarez & \\
\hline Lara & \\
\hline & \\
\hline nio Palomares & \\
\hline gorreta & \\
\hline Martín Lillo & \\
\hline .......... & \\
\hline érrez & \\
\hline Navales & \\
\hline arlos Mauricio & \\
\hline & \\
\hline & \\
\hline & \\
\hline
\end{tabular}

Agustín Moreno ................. 391

Víctor Cueto .................... 390

Ignacio Castaño .................. 390

Francisco Durán ................ 390

José García Meseguer .......... 390

Eduardo Saborido .................. 390

Juan Pedro Zamora .............. 390

Guillermo Saldaña ............... 390

José Barragán ................... 390

Anselmo Hoyos ................. 390

Francisco Martínez López ......... 390

Manuel Amor Deus .............. 389

Antonio de las Casas ........... 389

José María González Jerez ..... 389

Javier Terriente ................ 388

José Antonio Nieto ............ 388

María Salce Elvira ................ 388

Josep Palau ....................... 388

Emilio García López ............. 387

Joaquín Jordán ................... 387

Andreu Claret .................. 387

Rafael Pillado ................... 387

José María Carballido ........... 386

José Soriano ...................... 386

Lorenzo Hernández ............... 385

Manuel Vallejo .................... 385

Vicente Gutiérrez Solís .......... 385

Juan Francisco Pla ............. 385

Salvador Fuentes ................. 385

Angel Guerreiro ................ 384

Leopoldo Alcaraz ................ 383

Emilio Huertas .................. 383

Francisco Rodríguez ............ 382

Fuente: Mundo Obrero, 23-29 de diciembre de 1983.

\section{TABLA V \\ MIEMBROS NATOS DEL COMITE CENTRAL *}

Felipe Alcaraz Masats

J. Antonio Arrambarri

Adolfo Burriel

Angel Cristóbal

José Antonio García Rubio

Fernando González

Ignacio Latierro Corta

Ignacio Mantecón
Manuel Parejo

Julio Pérez de la Fuente

Adolfo Piñedo Simal

Pedro Antonio Ríos Martínez

Francisco Javier Suárez

José Valero González

Juan Villalba.

* Son miembros natos los secretarios políticos de nacionalidad y región.

FUENTE: Mundo Obrero, 23-29 de diciembre de 1983. 